\title{
Angle-Aware Greedy Forwarding Algorithm for Vehicular Ad Hoc Networks
}

\author{
Aye Zarchi Minn ${ }^{1}$ and May Zin $\mathrm{Oo}^{2}$ \\ Department of Computer Engineering and Information Technology, \\ Mandalay Technological University, Mandalay, Myanmar \\ Email: ${ }^{1}$ ayemin.rose@gmail.com, ${ }^{2}$ mayzinoo.mz@gmail.com
}

\begin{abstract}
Greedy Perimeter Stateless Routing (GPSR) gives good forwarding nodes for Mobile Ad Hoc Networks (MANET) but does not provide the right directional forwarding nodes in Vehicular Ad Hoc Network (VANET). This is the fact that MANET uses the nodes without limitations in moving but VANET uses fixed infrastructure in mobility such as roads with lanes. The key of original greedy forwarding is to choose the forwarding node with the shortest distance between the source and the destination. As it does not consider the direction of the forwarding nodes, it wrongly selects a forwarding node, which is going in the opposite direction from the destination. Thus, This paper tries to improve the greedy forwarding method, called angle-aware greedy forwarding algorithm of GPSR by taking into considerations in both the distance and the direction. Moreover, it shows the mystery of angle that gives the right direction. The optimized GPSR (OGPSR) using angle-aware greedy forwarding provides the better throughput, average end-to-end delay and routing overheads than the original GPSR.
\end{abstract}

Keywords: GPSR, Improved Greedy Forwarding Method, MANET, OGPSR, Original Greedy Forwarding Method, VANET.

\section{INTRODUCTION}

As VANET becomes popular for providing traffic information, accidents and weather conditions, there are a colossal number of researches trying to apply Ad Hoc Networks to VANET with increasing the number of vehicles and the complexity of towns-roads. However, the routing algorithms for VANET face many challenges because of the energetic topology, strict-delay requirements, high-speed moving, route obviousness, imprecise energy and accurate positioning.

For these reasons, routing protocols for MANETs have been investigated to suit to VANETs. Among them, position-based routing protocol fit in VANET having Global Positioning System (GPS) or higher armed forces supplied by Geographic Information System (GIS). Thus, they have become more and more accommodating in VANET.

There are many positioned based routing protocols. Geographic Source Routing (GSR) protocol [1] is aimed at urban roads where packets are forwarded to the destination by the shortest path from the resource to the goal in the digital plot using Dijkstra's algorithm. As GSR does not consider the junction for city scenarios, Greedy Perimeter coordinate Routing (GPCR) [2] finds a forwarding node in the junction, but not across the junction for the city scenarios. GPSR $[3,4]$ routing protocol is not only based on position but also an extremely suitable for VANETs. In GPSR, each vehicle knows its position via GPS or other location based technique. Every vehicle broadcasts a beacon message to one hop neighbors at userdefined time. At this time, all the devices will update their neighbor tables by storing the 
identification number of the message in its neighbor table. When required to send the message from the source to the destination, GPSR uses two forwarding strategies. Firstly, the packets are forwarded using the greedy forwarding strategy to find the forwarding node with the nearest distance to the destination. If there is no such node to be forwarded, then a recovering strategy is provided by using the Right-Hand rule called the perimeter forwarding strategy. It has relative multifaceted process to solve the problem of greedy.

In the perimeter forwarding, a Relative Naive Graph (RNG) or Garbie Graph (GG) is constructed and a line connecting the node that fails the greedy and destination node is implemented. By using the right-hand rule and face-change algorithm, the nodes located in the right of the line are chosen as the forwarding nodes. When the distance from the next hop node to the destination node is shorter than that from the greedy node and the destination node, GPSR ends the perimeter state and restarts the greedy state.

To utilize GPSR in VANET, it needs to be optimized to have better throughput, delay and routing overhead. The position-based routing protocols use greedy forwarding. It is often limited because direct communications may fail due to the incorrect moving in city roads of VANET. Previous researchers tried to improve GPSR summing the additional aids such as using digital maps to define junctions, anchors and streets and adding parameters for some constant values (velocity, direction). Therefore, this paper proposes a simple greedy forwarding method that takes the right direction using the arc of a tangent value.

\section{RELATED WORKS}

Many researches take into account many parameters such as the direction, speed and density of the nodes to improve the original greedy forwarding. It is also an advantage for VANET rather than MANET because MANET does not have finite routes for moving the nodes.

Author [5] proposed Greedy Perimeter Stateless Routing with Movement Awareness (GPSR-MA) by considering the speed, distance and the movement of moving vehicles for a routing decision. It underlines a gifted and forceful basic for automotive scenarios. However, it needs the history of node's coordinates to derive speed and direction.

A new mechanism [6] is proposed for upgrading the route selection of the Greedy mode of GPSR. It utilizes the distance and triangle area by the relay vehicles and implements a formula by adding two utilizations to determine the forwarding node. It outputs the better delay than GPSR. However, it does not provide a remarkable improvement in the ratio of packet delivery.

Moving Directional Based Greedy (MDBG) Routing Algorithm [7] is based on direction and used to develop routing selection in greedy. To obtain the definition of direction, it uses the hello messages, destination request messages and destination reply message. It can only solve the directional problem in greedy when source and target vehicle move far apart in opposite directions.

The technique presented in [8] designs a special Hello Packet with priority flag, such as vehicle moving course, velocity, concentration, and etc to assure effective route. When GPSR encounters the local maxima, it uses into the recovery scheme by buffering the prior data and recalculating the forwarding route. However, it does not consider the reduction of delay.

Link Quality and Velocity Vector GPSR (LQ-VV-GPSR) [9] selects realistic relay node to forward message wisely. Velocity vector and link status are utilized to achieve the balanced performance. But it is not be able to decrease routing overhead.

A routing protocol considering vehicles' density, as GPSR-MA moving direction and speed into GPSR to decide packet forwarding is described in [10]. Dissimilar GPSR-MA, it implements routing strategy with the consideration of movement and speed. The improved GPSR protocol has better performance in packet delivery rate, average throughput and end-toend delay under the urban simulation scenario. However it cannot resolve when the right forwarding node is positioned in the dissimilar direction of the target.

Our proposed system tries to solve the problem in greedy to choose a forwarding node in a right direction with a very simple arc of tangent rule. Optimized GPSR (OGPSR) Protocol is 
also proposed to improve greedy forwarding in GPSR together with the mystery of the arc of tangent as a parameter to determine the right forwarding from the features of VANET.

\section{OGPSR PROTOCOL WITH IMPROVED GREEDY FORWARDING ALGORITHM}

As shown in Fig. 1, a vehicle is represented as a triangle and the tip of the triangle represents the direction of the vehicle. All the vehicles move along the road with two directions. Assume that $\mathrm{S}$ wants to send a data packet to node $\mathrm{D}$ at time t. The vehicle $\mathrm{S}$ has five neighbors in which $\mathrm{E}, \mathrm{F}$ and $\mathrm{G}$ will be at a shorter distance than that from S to D. GPSR chooses $\mathrm{F}$ to forward the packet using greedy forwarding algorithm. However, node F is moving away from node D. Ultimately, the data packet carried by node F will be discarded at the next time. Therefore, without considering direction of the movements, the GPSR protocol can cause errors such as wrong packet forwarding, long time in transmissions and high routing overhead.

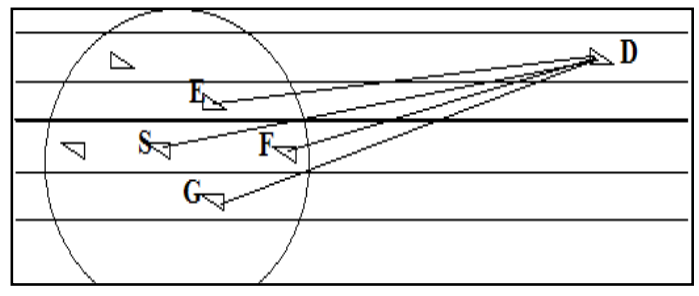

FIGURE 1: Wrong forwarding of original GPSR in VANET.

The key point of greedy forwarding is to seek the forwarding nodes depending only on the distance towards reaching the destination. Therefore, it cannot always provide correct results for moving nodes. As such, the direction becomes the second key point to improve greedy. OGPSR using improved greedy proposes that the right direction can be given only with the arc of tangent value under VANET architecture.

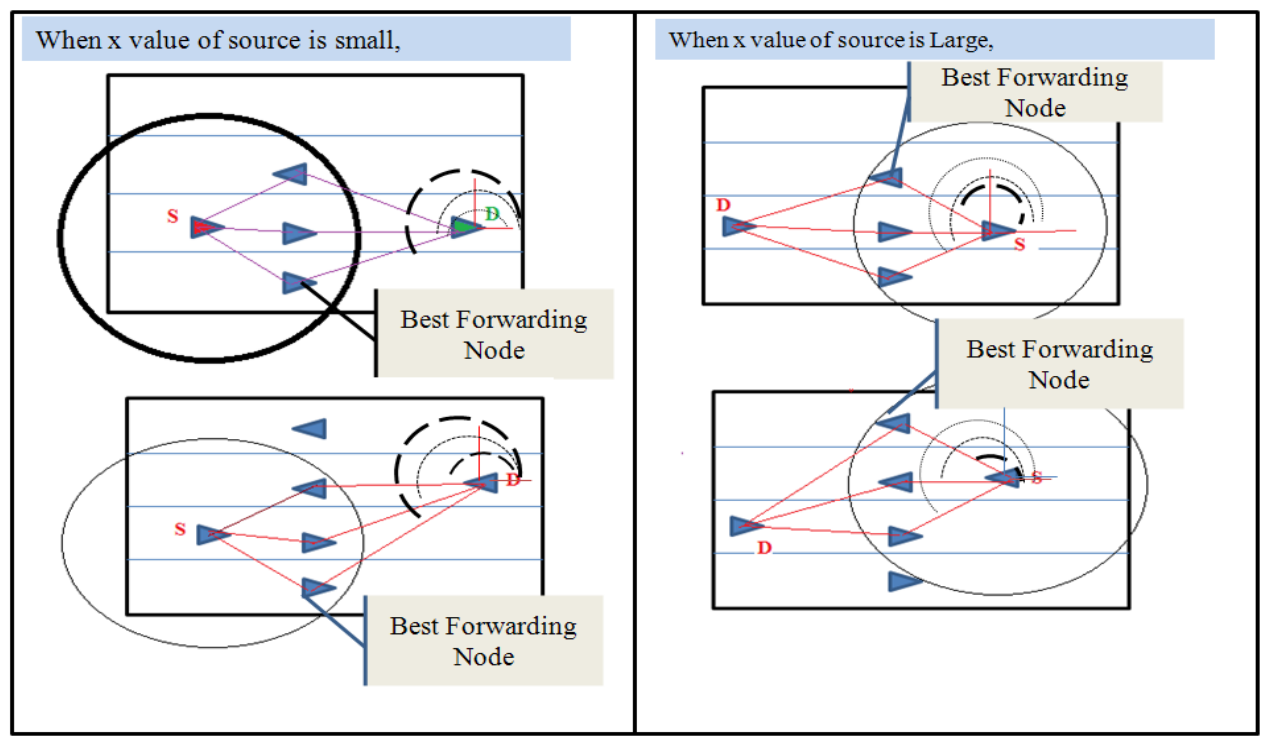

FIGURE 2: Finding the best forwarding node for horizontal road.

Supposing all vehicles are fixed with a GPS machine, the system assumes that each node can gain geographical information provided by the GPS. Considering the requirements of the GPSR protocol, this paper proposes an optimized GPSR protocol by designing an improved greedy forwarding specialized for VANET to overcome the challenges illustrated in Fig. 1.

OGPSR is based on the structures of the roads with lanes in VANET to choose the right 
direction. It takes into consideration on horizontal and vertical roads by assuming that all roads have two lanes.

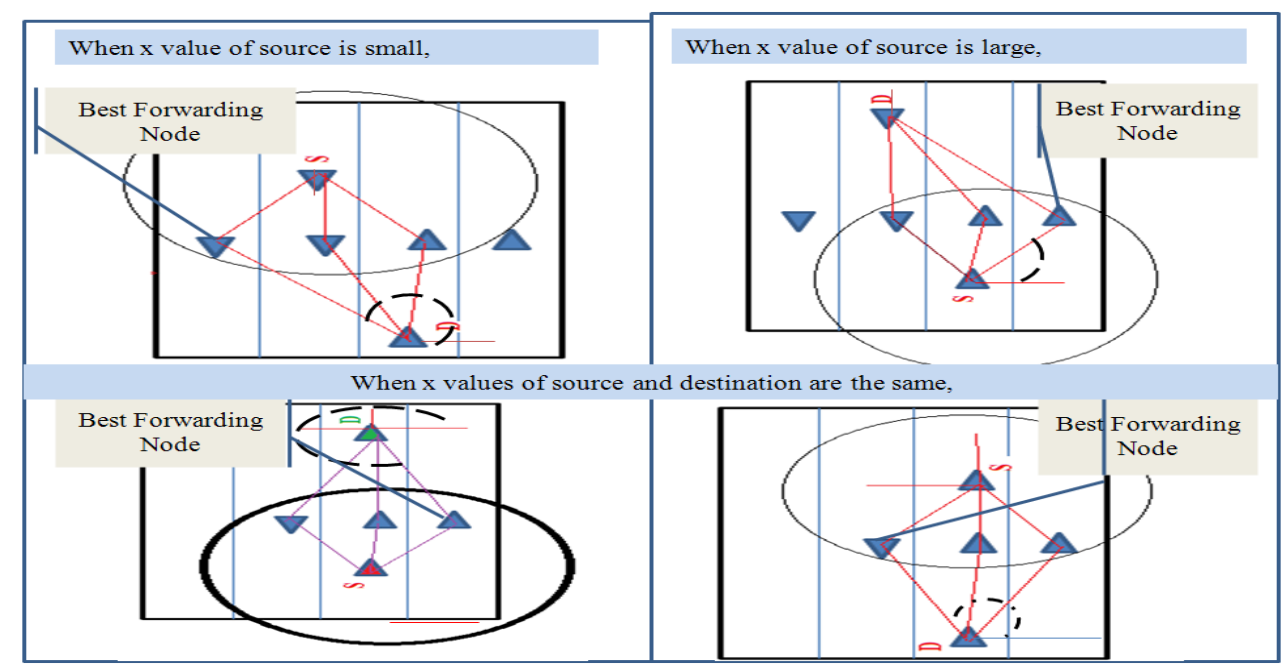

FIGURE 3: Finding the best forwarding node for vertical road.

The left side of the Fig. 2 is the first consideration in which $\mathrm{x}$ coordinate value of source is smaller than that of destination. On the other hand, the right side is the second consideration, in which $\mathrm{x}$ coordinate value of destination is larger than that of source. For the left side, a neighboring node of the source $\mathrm{S}$ with the largest arc of tangent value from $\mathrm{x}$ coordinate of the destination point represents the best forwarding node to correctly direct to the destination node $\mathrm{D}$ at the next time. The best result for the right side is selecting the forwarding node with the smallest arc of tangent value from $\mathrm{x}$ coordinate of the source point.

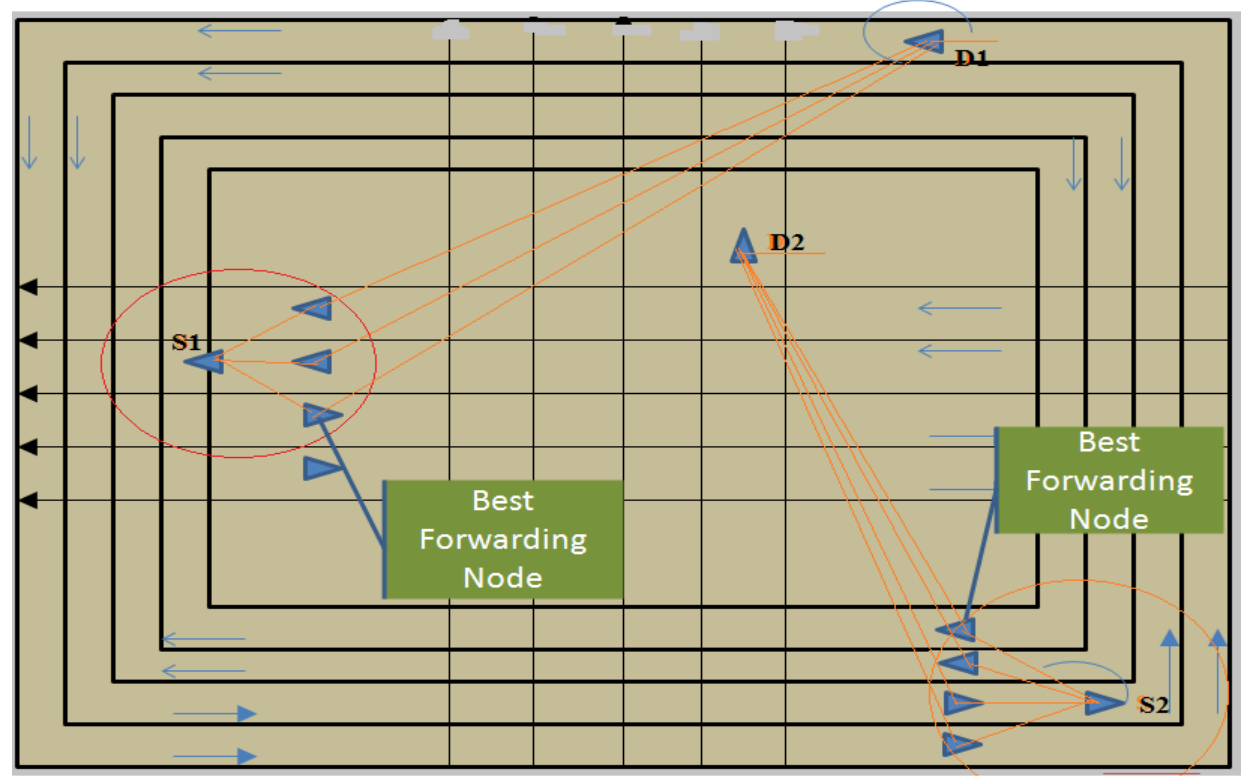

FIGURE 4: Finding the best forwarding node for maps with horizontal and vertical roads.

The two considerations described in horizontal road can be seen the upper part of vertical road shown in Fig. 3. The lower part of the figure gives the third consideration having the same $\mathrm{x}$ coordinates value in both source and destination nodes. In the lower part of the figure, the node with the largest arc of tan value from $\mathrm{x}$ coordinate of the destination point will be the best forwarding node. 
Fig. 4 shows a map with the horizontal and vertical roads together and it can be found that the previous considerations can give the best forwarding node for this map. For the source S1 and destination D1, the $\mathrm{x}$ value of source is smaller than that of destination and thus, the right direction can be given with the largest atan 2 angle from the point D1. The selected forwarding node leads to the destination for the next time. Similarly, the node S2 can choose the best forwarding node with the leading direction to D2.

Unlike GPSR, OGPSR has the two key points (distance and direction). For the distance key, the forwarding node has a shorter distance than the source from the destination. For the direction key, Table 1 is constructed with decisions based on three considerations.

TABLE 1: Decision rules for direction metrics.

\begin{tabular}{l|l|l} 
Cases & \multicolumn{1}{|c|}{ Considerations } & \multicolumn{1}{|c}{ Decision Rules } \\
\hline I & $\begin{array}{l}\text { When the } \mathrm{x} \text { coordinate of the } \\
\text { source is smaller than that of the } \\
\text { destination, }\end{array}$ & $\begin{array}{l}\text { the best forwarding node is with the } \\
\text { largest arc of tangent value from } \mathrm{x} \\
\text { coordinate for the destination. }\end{array}$ \\
II & $\begin{array}{l}\text { When the } \mathrm{x} \text { coordinate of the } \\
\text { source is larger than that of the } \\
\text { destination, }\end{array}$ & $\begin{array}{l}\text { lhe best forwarding node is with the } \\
\text { smallest arc of tangent value from } \mathrm{x} \\
\text { coordinate for the source. }\end{array}$ \\
$\begin{array}{l}\text { When the } \mathrm{x} \text { coordinates for the } \\
\text { source and destination are the } \\
\text { same, }\end{array}$ & $\begin{array}{l}\text { largest arc of tangent value from } \mathrm{x} \\
\text { coordinate for the destination. }\end{array}$
\end{tabular}

The following algorithm is implemented to improve greedy forwarding using decision rules in OGPSR.

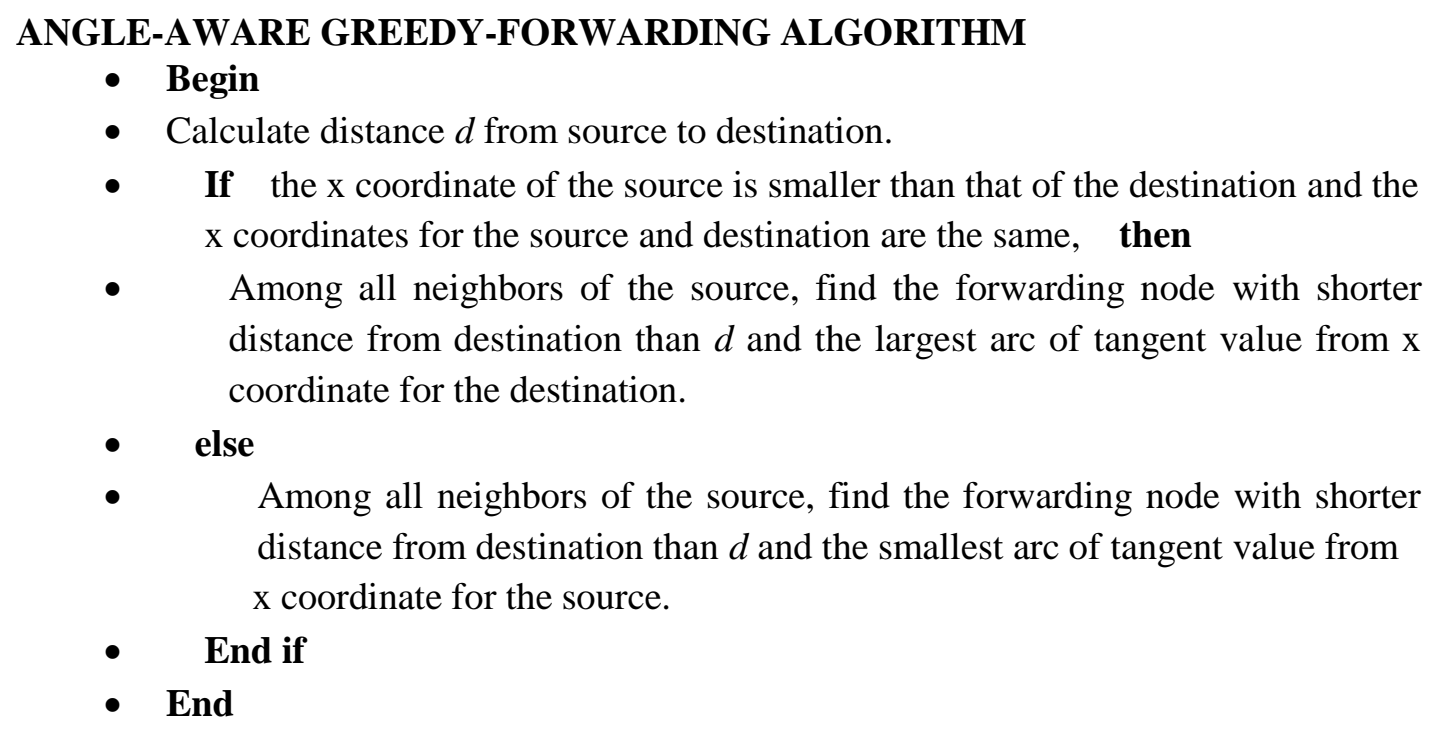

OGPSR is the same perimeter forwarding function in GPSR. It does not change the procedures in that of GPSR. It uses a constant beacon update interval as original GPSR.

\section{RESULTS AND DISCUSSION}

Simulation is carried out in Network Simulator-2 (NS-2) [11] running on Ubuntu System. Manhattan and Freeway models $[12,13]$ are used for mobility. Manhattan model can give the urban maps crossing horizontal and vertical streets using a cross-road topology. There are 
two-way roads without lanes. It also provides setting the vehicles' velocity. Freeway is for highway scenario that supports two-way roads with lanes. The simulations are run with the following parameter settings as in Table 2 .

TABLE 2: Simulation Environment Parameters.

\begin{tabular}{l|l}
\multicolumn{1}{c|}{ Parameter Name } & \multicolumn{1}{c}{ Value } \\
\hline Scenario size & $500 \mathrm{~m} \times 500 \mathrm{~m}$ \\
MAC & IEEE 802.11 \\
Antenna type & Omni-Antenna \\
Traffic type & CBR \\
Mobility models & HW, MH \\
Node velocity & $20 \mathrm{~m} / \mathrm{s}$ for max, $10 \mathrm{~m} / \mathrm{s}$ for min \\
Number of Nodes & $50,75,100,125 \mathrm{modes}$ \\
Simulation Time & 100 seconds \\
Propagation & Two way Ground \\
Packet size & 512 bytes \\
Beacon Update Interval & 1 second
\end{tabular}

\subsection{Measurements for Urban Maps}

As a performance testing, throughput, average end-to-end delay and routing overhead are compared with different number of vehicles on urban maps as shown in Fig. 5. The throughput values of OGPSR are not very significant when compared to GPSR. However, it is found that OGPSR outperforms when measuring average end-to-end delay. This is because OGPSR can forward right directional forwarding nodes and reduce delays. Moreover, OGPSR gives less routing overhead than original protocol. OGPSR reduces $13.7 \%$ in average delay and $3.58 \%$ in routing overhead.

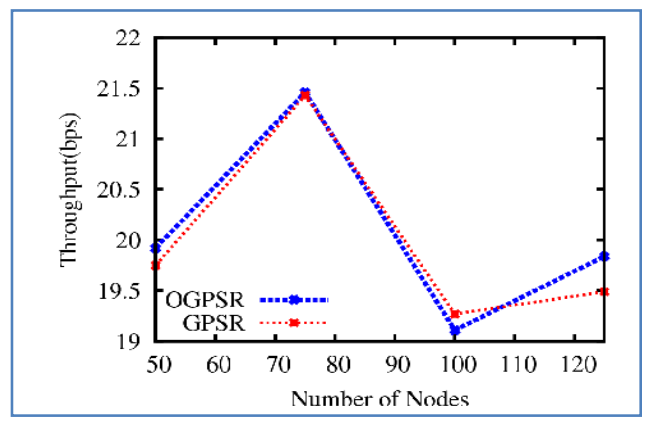

(a)

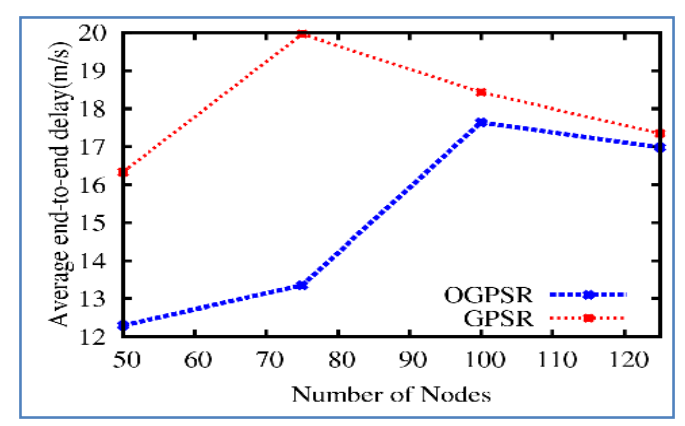

(b)

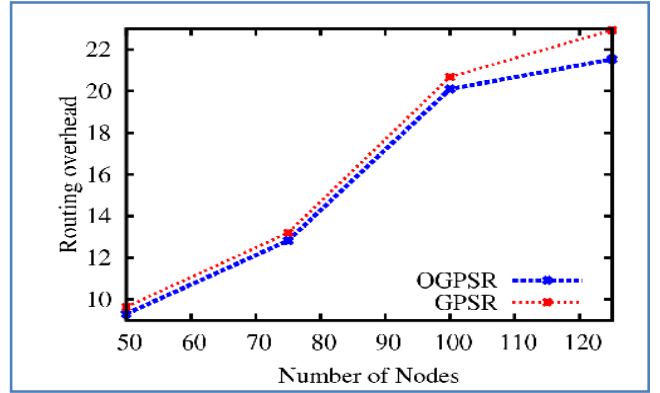

(c)

FIGURE 5: Performance comparison between OGPSR and GPSR for urban maps (a) Throughput Measurement, (b) Average end-to-end Delay Measurement and (c) Routing Overhead Measurement. 


\subsection{Measurement for Highway Maps}

For the highway maps in Fig. 6, considering throughput, average end-to-end delay and routing overhead with the variation of the node density, OGPSR gives the better results for all scenarios. Not only throughput in OGPSR outperforms GPSR but also OGPSR produces better throughput, lower average end-to-end delay and routing overhead than GPSR.

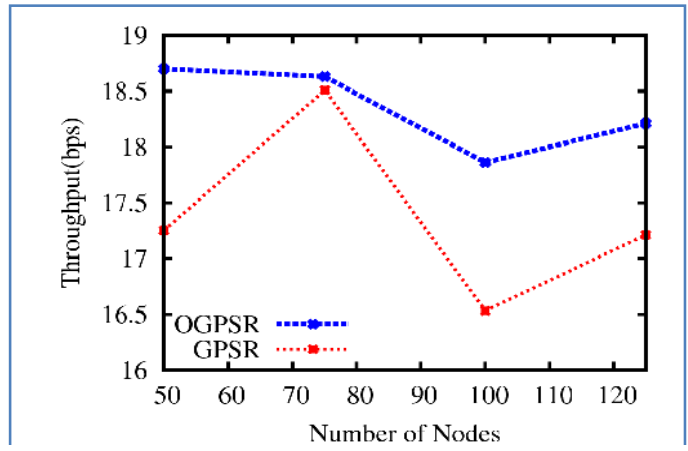

(a)

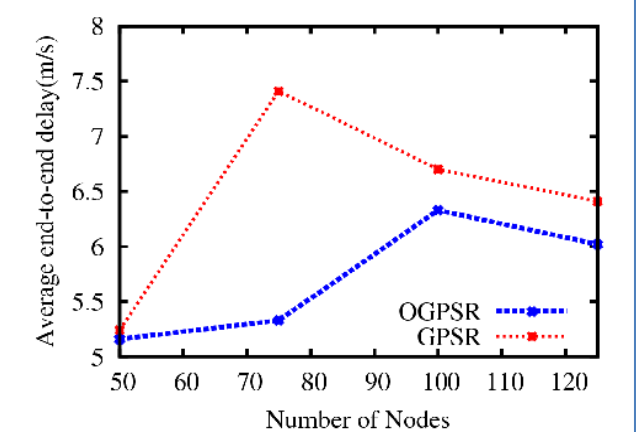

(b)

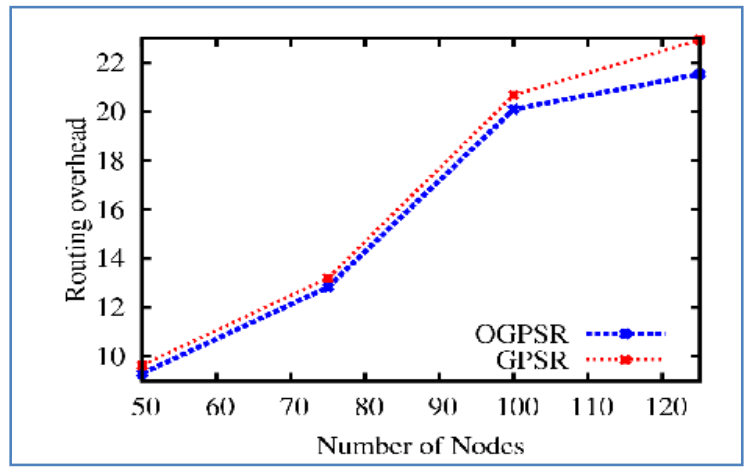

(c)

FIGURE 6: Performance comparison between OGPSR and GPSR for highway maps

(a) Throughput Measurement, (b) Average end-to-end Delay Measurement and (c) Routing Overhead Measurement.

\section{CONSLUSION \& FUTURE WORK}

In this paper, improved greedy forwarding method is proposed for optimizing GPSR. Original and optimized GPSR protocols are simulated on urban and highway maps for vehicular ad hoc networks. OGPSR is designed for horizontal and vertical lanes as similar as highway. Hence, it can be said that it is more suitable in highways because OGPSR can give better results in a highway than in an urban. For urban roads, simulation results show that the throughput and the routing overhead performance of both protocols are not significantly different from each other whereas for highway maps, OGPSR can give a better throughput and lower routing overhead than GPSR. However, OGPSR outperforms GPSR in terms of average end-to-end delay while varying the number of vehicles for both mobility models. The main idea using VANET is sending important messages within vehicles in time. As a whole, it can be said that OGPSR is a good choice to optimize vehicular ad hoc network because it has good scalability and less delay. Future work is to find the angle calculation for the right direction in roads in real time.

\section{REFERENCES}

[1] C. Lochert, H. Hartenstein, J. Tian, et al., "A routing strategy for vehicular ad hoc network in the city environments", Proc. of IEEE Intelligent Vehicles Symposium, June 2003. 
[2] C. Lochert, M. Mauve, H. üßler, et al., "Geographic routing in city scenarios", Proc. of ACM SIGMOBILE Mobile Computing and Communication Review, January 2005.

[3] J. Bernsen, D. Manivannan, "Greedy routing protocols for vehicular ad hoc networks," in Proc. of the 7th International Conference on Wireless Communications and Mobile Computing (IWCMC), pp. 632-637, August 2008.

[4] B. Karp and H. T. Kung, "GPSR: greedy perimeter stateless routing for wireless network", Proc. of ACM/IEEE MobiCom, August 2000.

[5] Fabrizio Granelli, Giulia Boato, Dzmitry Kliazovich and Gianni Vernazza, "Enhanced GPSR Routing in Multi-Hop Vehicular Communications through Movement Awareness", IEEE Communications Letter, vol. 11, no. 10, pp. 781-783, 2007.

[6] Zhang Ning, Yunho Jung, Yan Jin, Kee-Cheon Kim, "Route Optimization for GPSR in VANET", 2009 IEEE International Advance Computing Conference (IACC 2009), Patiala, India, 6-7, March 2009.

[7] HungChin Jang and Hsiang-Te Huang, "Moving Direction Based Greedy Routing Algorithm for VANET", Computer Symposium (ICS) International Journal, Tainan, pp. 535-540, 2010.

[8] Lili Hu, Zhizhong Ding, Huijing Shi. "An Improved GPSR Routing Strategy in VANET", Wireless Communication, Networking and Mobile Computing (WiCOM), $8^{\text {th }}$ International Conference on Shanghai China, 2012.

[9] Hao Wang, Guo-zhen Tan and Jiziang Yang, "An Improved VANET Intelligent Forward Decision-making Routing Algorithm", Journal of Networks, vol.7, no.10, 2012.

[10] Degui Xiao, Lixiang Peng, Clement Ogugua Asogwa, Lei Huang, "An Improved GPSR Routing Protocol", International Journal of Advancements in Computing Technology, vol. 3, no. 5, 2011.

[11] The Network Simulator NS-2, http://www.isi.edu/nsnam/ns/index.html.

[12] Bai F., Sadagopan N., Helmy A, "Important: A framework to systematically analyze the impact of mobility on performance of routing protocols for ad hoc networks", In Proceedings of IEEE Societies Twenty-Second Annual Joint Conference of the IEEE Computer and Communications, pp. 825-835, 2003.

[13] Khairnar V. D., Pradhan S. N., "Mobility models for Vehicular Ad-hoc Network Simulation", In Proceedings of 2011 IEEE Symposium on Computers \& Informatics (ISCI), pp. 460-465, 2011.

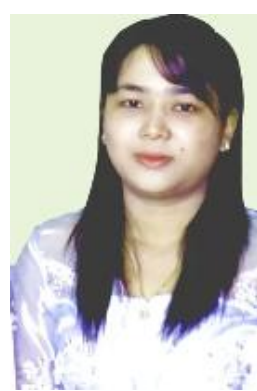

Aye Zarchi Minn was born in Bago, Myanmar, in 1982. She received the Bachelor of Engineering degree (B.E) in the department of Information Technology from the Technological University of Taunggyi,in 2005, and Master of Engineering (M.E) in the department of Information Technology from the Mandalay Technological University in 2007. She is currently a $\mathrm{Ph} . \mathrm{D}$. candidate in the department of Computer Engineering and Information Technology at Mandalay Technological University. From 2007 to 2014, she was a Lecturer in the department of Information Technology, Pyay Technological University, Myanmar. Presently, her research interest includes the development of routing protocols in mobile and vehicular applications related to computer network and protocols.

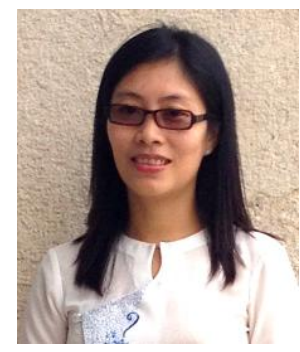

May Zin Oo is an associate professor at Mandalay Technological University, Myanmar. She received her first B.E. degree from Mandalay Technological University. Later she received her M.E. degree from Yangon Technological University. Afterwards, she obtained a Ph.D. degree in wireless communication from the University of Malaya, Malaysia in 2012. Her research interests include wireless communication and networking. 